\title{
Role of Helicobacter pylori in the triggering and evolution of hemorrhagic gastro-duodenal lesions
}

\author{
DRAGOS GEORGE POPA ${ }^{1}$, COSMIN VASILE OBLEAGA ${ }^{2 *}$, BOGDAN SOCEA ${ }^{3,4}$, DRAGOS SERBAN ${ }^{3,5}$, \\ MARIUS EUGEN CIUREA ${ }^{1}$, MARIAN DIACONESCU ${ }^{2}$, IONICĂ DANIEL VÎLCEA ${ }^{2}$, CRISTIAN MEȘINĂ ${ }^{2}$, \\ CECIL MIREA $^{2}$, DAN NICOLAE FLORESCU ${ }^{6}$, VLAD DUMITRU BALEANU ${ }^{6}$, MEDA COMANDASU ${ }^{5}$, \\ MIHAI SILVIU TUDOSIE ${ }^{3}$, LAURA CARINA TRIBUS ${ }^{3,7^{*}}$ and BOGDAN NICULESCU ${ }^{8}$
}

\begin{abstract}
Departments of ${ }^{1}$ Plastic and Reconstructive Surgery, and ${ }^{2}$ Surgery, Craiova University of Medicine and Pharmacy, 200349 Craiova; ${ }^{3}$ Faculty of Medicine, 'Carol Davila' University of Medicine and Pharmacy Bucharest, 020021 Bucharest; ${ }^{4}$ Department of General Surgery, 'Sf. Pantelimon' Clinical Emergency Hospital, 021659 Bucharest; ${ }^{5}$ Fourth Department of General Surgery, Emergency University Hospital Bucharest, 050098 Bucharest; ${ }^{6}$ Department of Gastroenterology, Craiova University of Medicine and Pharmacy, 200349 Craiova; ${ }^{7}$ Gastroenterology Department, Emergency University Hospital Bucharest;

${ }^{8}$ Department of Sports and Health, 'Constantin Brancusi' University, 210152 Targu Jiu, Romania
\end{abstract}

Received June 8, 2021; Accepted July 8, 2021

DOI: $10.3892 / \mathrm{etm} .2021 .10582$

\begin{abstract}
The majority of studies concerning Helicobacter pylori (H. pylori) are oriented towards the implication of infection with $H$. pylori in processes that end in the formation of neoplasia, without assessing the impact of the bacterium in triggering acute gastroduodenal hemorrhagic episodes. The present study includes 166 patients with upper digestive hemorrhage, admitted to the ATI Clinic, the Gastroenterology Clinic or to the Surgery II Clinic of the County Emergency Clinical Hospital in Craiova, Romania between 2017 and 2019. All patients were monitored for evolution and received treatment according to current guidelines, and hemorrhagic lesions were biopsied. In the study group, $56.8 \%$ of the patients with upper gastrointestinal bleeding (UGIB) were positive for $H$. pylori and $43.2 \%$ were negative. In patients less than 50 years of age, non-steroidal anti-inflammatory drug (NSAID) use and H. pylori infection had a cumulative effect in causing bleeding lesions, but in patients older than 50 years of age, the use of
\end{abstract}

Correspondence to: Dr Dragos Serban, Faculty of Medicine, 'Carol Davila' University of Medicine and Pharmacy Bucharest, 37 Dionisie Lupu Street, 020021 Bucharest, Romania

E-mail: dragos.serban@umfcd.ro

*Contributed equally

Abbreviations: UGIB, upper gastrointestinal bleeding; H. pylori, Helicobacter pylori; NSAIDs, non-steroidal anti-inflammatory drugs; IHC, immunohistochemical examination; MALT, gastric mucosa-associated lymphoma

Key words: Helicobacter pylori, upper gastrointestinal bleeding, endoscopic hemostasis, surgery, anticoagulant agents, neoplasia
NSAIDs was replaced by therapies with oral antiplatelet or anticoagulant agents. The need for hemostasis surgery was more common in patients who exhibited $H$. pylori-positive UGIB compared to $H$. pylori-negative (16 vs. $9.7 \%$ ). In patients with $H$. pylori-positive hemorrhagic lesions, gastric resection was frequently required to obtain hemostasis. Persistence of H. pylori infection in patients with a history of gastric resection $(4.1 \%)$ still predisposes to a hemorrhagic or neoplastic complication.

\section{Introduction}

Marked progress has been made in the management of non-varicose upper gastrointestinal bleeding (UGIB) due to the introduction of drugs that decrease gastric acid secretion, Helicobacter pylori (H. pylori) eradication therapies, and improved endoscopic hemostasis, yet, the mortality rate has remained relatively constant (1). H. pylori induces chronic superficial gastritis with neutrophil infiltration into the mucosa, therefore, it has been speculated that $H$. pylori infection underlies the bleeding lesion (2). Gastric and duodenal ulcers are strongly related to $H$. pylori (2). In initial reports around the world, in the first decade after the discovery of H. pylori, $\sim 95 \%$ of duodenal ulcers and $85 \%$ of gastric ulcers were associated with $H$. pylori infection (3), and eradication of $H$. pylori changed the natural course of ulcer disease and almost completely prevented the recurrence of the ulcer (4). Hemorrhage is the most common complication of ulcer disease and is estimated to be present in $15-20 \%$ of ulcers. Approximately $40 \%$ of patients with UGIB have a hemorrhagic ulcer, and ulcerative disease is therefore the leading cause of upper gastrointestinal hemorrhage (1). Eradication of $H$. pylori greatly reduces the risk of ulcer and also bleeding in those patients whose hemorrhage was due to H.pylori infection (4). Mortality varies between 3 and 14\% and has not 
changed in the last 10 years $(1,4)$. Mortality increases with age and is significantly higher in patients who are already hospitalized for associated comorbidities. Risk factors for bleeding in peptic ulcer are the administration of non-steroidal anti-inflammatory drugs (NSAIDs), oral anticoagulants and $H$. pylori infection. $H$. pylori infection increases the risk of UGIB by 1.7 times, but the exact influence of $H$. pylori in the evolution of UGIB (spontaneous hemostasis or to surgery) is not known $(5,6)$.

The present study aimed to analyze the factors of care influencing the evolution of non-variceal UGIB dating some lesions caused by $H$. pylori infection, as well as to assess the relationship between bacteria and alcohol consumption, NSAIDs and oral antiplatelet/anticoagulated agents in triggering lesion hemorrhage.

\section{Patients and methods}

The study included 166 patients with clinical signs of UGIB, hospitalized in the ATI Clinic, Gastroenterology Clinic or in the Surgery II Clinic of the County Emergency Clinical Hospital (SCJU) in Craiova, Romania between 2017 and 2019 (3 years). The diagnosis of non-variceal UGIB was established by objective clinical examination and endoscopic examination, and the diagnosis of $H$. pylori infection was made by noninvasive tests and by histological and immunohistochemical examination (IHC). Testing for H. pylori was conducted in most patients with signs of UGIB after stopping the bleeding episode. Patients were tested for $H$. pylori using fecal antigen detection (after melena termination, normal-looking stool) and by highlighting this bacterium by specific histological staining (performed from gastric endobiopsies or surgery). Biopsy was collected during the first endoscopy, after hemostasis therapy, in patients without massive bleeding and without the diagnosis of $H$. pylori infection prior to the hemorrhagic episode. The biopsy was obtained from the gastric mucosa from 5 different sites, according to the Updated Sydney System (7): 2 from the antrum, 2 from the mucosa of the gastric body and one from the gastric incision. In the case of suspicious or certain lesions for malignant lesions, several biopsies were taken from this level. Two types of PENTAX EG-290 and EVIS EXERA III Olympus endoscopy devices were used for endoscopic therapy and biopsy collection. The bioptic samples were processed in the Pathological Anatomy Laboratory of the SCJU in Craiova and in the Center for Studies of Microscopic Morphology and Immunology within the University of Medicine and Pharmacy in Craiova, Romania. The stains used to highlight the bacterium were hematoxylin and eosin and Giemsa. IHC has superior accuracy in highlighting $H$. pylori from gastric biopsy collected from patients with non-variceal UGIB who were both positive and negative on the noninvasive test.

Histological evaluation of endobiopsies using common stains is considered the 'gold standard' in identifying this bacterium. The samples obtained were collected in containers with formalin solution and were processed according to the standard technique of paraffin inclusion, following the following steps: fixation in $10 \%$ buffered formalin, washing with water or $80 \%$ alcohol, dehydration (by successive alcohol baths), clarification (by baths of benzene, toluene, xylene and paraffin). The final results were obtained after an interval of 20-25 days from the sampling of the biopsy fragments. At 30 days after discharge, patients were informed of the histological and IHC results performed from biopsies collected at endoscopic evaluation or by open surgery (in cases where hemostasis was obtained surgically). Another purpose for the introduction of routine IHC was related to the evaluation of the value of this protocol in the diagnosis of subsequent or associated lesions: bleeding: acute/chronic gastritis, metaplasia, cancer or gastric lymphoma. Although histological examination is accurate in providing data on the degree of atrophy, metaphase, or carcinoma, the addition of IHC examination provides additional information and establishes the correct diagnosis in all cases. The use of IHC was available: the LSAB (HRP) method [labeled streptavidin biotin (LSAB) and horseradish peroxidase (HRP)] and anti-H. pylori antibodies were used.

The evolution of patients was analyzed in terms of: bleeding rate, the presence of other risk factors, the need for surgical treatment, and the length of hospitalization (influenced by surgery). Eradication of $H$. pylori infection was defined as negative results for the fecal antigen testing after specific therapy. We decided to retest for $H$. pylori for all patients tested, regardless of whether the initial result was positive or negative. Retesting was performed after discontinuation of proton pump inhibitors. Testing for $H$. pylori was performed in patients with clinical signs of non-variceal UGIB without a previous history of bleeding.

\section{Results}

Testing for $H$. pylori was consistently positive in both the noninvasive test and the histological evaluation, respectively IHC. After histological evaluation and IHC, of the 166 patients tested for $H$. pylori and studied, group A consisted of $96 \mathrm{H}$. pylori-positive patients and group B of 70 $H$. pylori-negative patients. At 30 days after discharge, all patients were retested. Of the 76 initially negative patients, $70(92.1 \%)$ remained negative, and 6 patients $(7.9 \%)$ became positive (histological examination and IHC confirmed their positivity for $H$. pylori). Of the 96 initially positive patients who received specific treatment, 20 remained positive and 70 patients became negative.

Treatment of $H$. pylori infection was initiated during hospitalization, immediately after the bleeding stopped and continued in the outpatient setting. All patients received treatment with omeprazole $20 \mathrm{mg}$ twice daily, amoxicillin $1 \mathrm{~g}$ twice daily and clarithromycin $500 \mathrm{mg}$ twice daily. We decided on this treatment scheme for several reasons: i) clarithromycin and amoxicillin have a low rate of prescription in this area and thus there is a low rate of resistance; ii) it is an easy treatment scheme with minimal side effects; and iv) is easy to administer. In order to prevent possible treatment discontinuation, patients were asked about the side effects of the therapy and we were assured of the correct follow-up of this treatment. Evaluation of resistance to first-line therapy of $H$. pylori was performed using the non-invasive test used at diagnosis (detection of specific antigen in feces). All patients resistant to first-line therapy ( $22 \%$ of total) were re-evaluated endoscopically and received second-line therapy. 


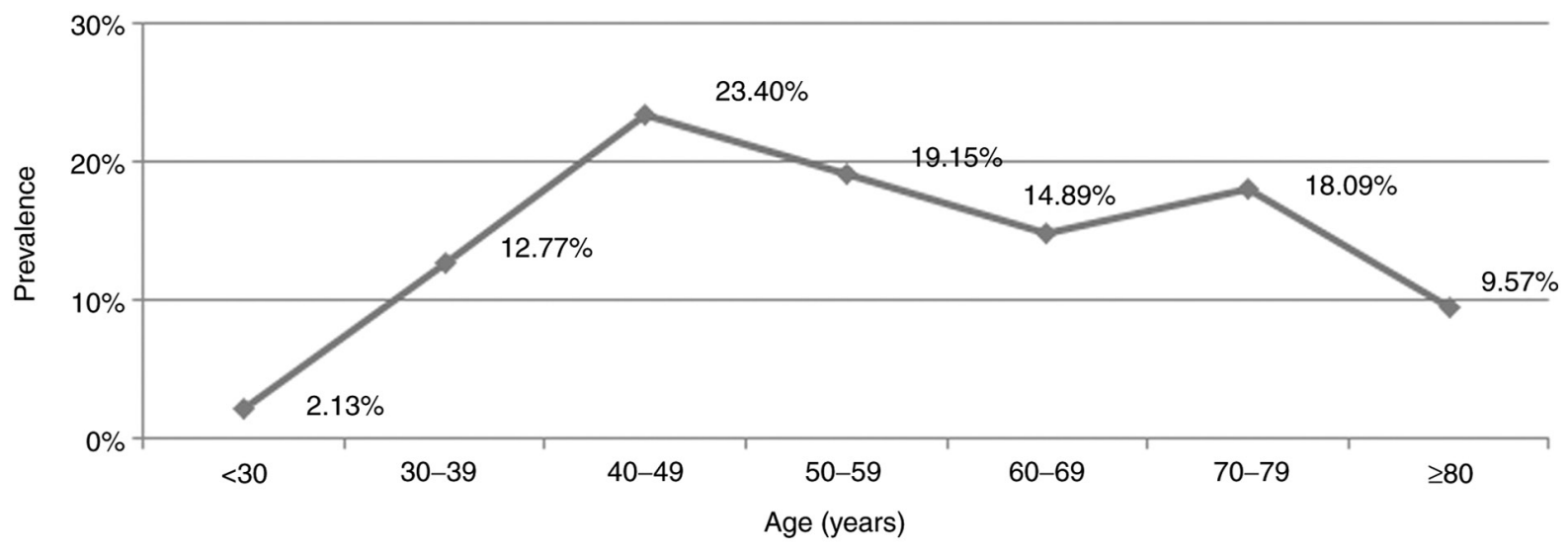

Figure 1. Prevalence of $H$. pylori-positive hemorrhagic lesions in the studied group according to age (in years). H.pylori, Helicobacter pylori.

The second non-invasive test provided information on identifying 6 more patients who at the first test were false-negative as well as data on the resistance of the bacterium in the cases initially treated. We also included in the study 4 patients with a history of gastric resection for gastric ulcer. Endoscopic evaluation revealed acute anastomotic ulcer and endoscopic hemostasis was effective.

There was an increased frequency of hemorrhagic lesions in $H$. pylori-positive men compared to women with the same lesions ( 84.04 vs. $15.96 \%)$ In the $H$. pylori-negative group, there was also a male prevalence, but with a lower M:F sex ratio (71.83 vs. $29.17 \%)$.

The presence of $H$. pylori was increased in the studied group directly proportional to age $\leq 50$ years, and then decreased slightly in elder age. The most frequent hemorrhagic lesions caused by $H$. pylori were in the age segment between 40 and 50 years. In this age segment, $>23 \%$ of non-variceal UGIB were directly related to the presence of H. pylori (Fig. 1).

The relationship between NSAID use and $H$. pylori infection in patients $<50$ years of age had a cumulative effect in causing bleeding. Therefore, the main causes of lesion bleeding were $H$. pylori infection and NSAID use. After the age of 50 years, the main causes included H.pylori infection and treatment with antiplatelet agents/oral anticoagulants. In the group of $H$. pylori-positive patients, $56.5 \%$ of patients $<50$ years of age stated that they had consumed NSAIDs for $>5$ days, compared to only $16.67 \%$ of $H$. pylori-negative patients $>50$ years of age. Therapies with antiplatelet agents or oral anticoagulants were the direct causes of UGIB in $45 \%$ of patients with $H$. pylori-positive gastro-duodenal lesions $>50$ years of age. The evolution of a non-variceal UGIB can be significantly influenced by the value of coagulation times (modifications of these therapies), at the same time the transfusion requirement is higher in these patients, regardless of the presence of $H$. pylori (Table I).

In elderly patients with associated comorbidities, an episode of UGIB can be fatal. We analyzed the effect of NSAID use associated with alcohol consumption in patients with hemorrhagic lesions, depending on the presence of $H$.pylori and found the following: $28.75 \%$ of $\mathrm{H}$.pylori-positive patients with active bleeding at endoscopic evaluation (Forrest
Table I. Relationship between NSAID use and age-related anticoagulants/antiplatelet agents in $\mathrm{H}$. pylori-positive patients.

\begin{tabular}{lcc}
\hline Age (years) & $<50(\%)$ & $>50(\%)$ \\
\hline NSAIDs & 56.52 & 16.67 \\
Anticoagulants & 4.35 & 45 \\
No treatment & 39.13 & 38.33 \\
\hline
\end{tabular}

H. pylori, Helicobacter pylori; NSAIDs, non-steroidal anti-inflammatory drugs.

class IA and IB) were treated with NSAIDs and consumed alcohol chronically compared to $12.50 \%$ of $H$. pylori-negative patients (Table II).

Endoscopic evaluation (early or late) was performed in all patients tested for $H$. pylori who had hemorrhagic lesions and we classified the gastric lesions found on endoscopy as follows: active bleeding requiring endoscopic or surgical hemostasis (endoscopic bleeding) and no active bleeding-supervision. Lesions with active bleeding were classified as follows: hemorrhagic gastroduodenitis (petechiae and erosions of the gastroduodenal mucosa), acute gastric ulcer, chronic gastric ulcer, acute duodenal ulcer, chronic duodenal ulcer and gastric tumor. We also classified the lesions without active bleeding. Endoscopic features have been reported to be useful tools for diagnosing $H$. pylori infection in the gastric mucosa. However, the ability to predict these characteristics can vary greatly (8) (Table III).

Acute hemorrhagic lesions (gastric, duodenal ulcer or hemorrhagic gastroduodenitis) were detected more frequently in the group of positive than negative $H$. pylori patients (69 vs. 43 cases). At the same time, the rate of acute lesions in the stomach and duodenum was higher in $H$. pylori-positive patients. Chronic hemorrhagic or non-hemorrhagic gastric ulcer was found in similar proportions in both the negative and positive patients. Hemorrhagic gastroduodenitis lesions were found in equal proportions in the studied groups. Evaluation of patients with active bleeding on endoscopic examination showed that the bleeding rate was high in the H.pylori-positive patients who were negative after specific therapy (18.18\%) compared to those with $H$. pylori-negative lesions (15.79\%) 
Table II. Determinants of bleeding depending on the presence of H.pylori.

\begin{tabular}{|c|c|c|c|}
\hline & $\begin{array}{c}\text { H.pylori-negative, } \\
\text { with NSAID + alcohol }(\%)\end{array}$ & $\begin{array}{c}\text { H.pylori-positive, } \\
\text { without NSAID + alcohol }(\%)\end{array}$ & $\begin{array}{c}\text { H. pylori-positive, } \\
\text { with NSAID + alcohol }(\%)\end{array}$ \\
\hline Active bleeding lesions & 12.5 & 14.29 & 28.75 \\
\hline No active bleeding lesions & 87.5 & 85.71 & 71.25 \\
\hline
\end{tabular}

H. pylori, Helicobacter pylori; NSAID, non-steroidal anti-inflammatory drugs.

Table III. Endoscopic evaluation of H.pylori-positive vs. H. pylori-negative patients.

H.pylori-negative

H.pylori-positive

Hemorrhagic acute duodenal ulcer 6

Non-hemorrhagic acute duodenal ulcer

Hemorrhagic chronic duodenal ulcer

9

9

Hemorrhagic chronic duodenal ulcer

11

6

Hemorrhagic acute gastric ulcer

6

Non-hemorrhagic acute gastric ulcer

Hemorrhagic chronic gastric ulcer

9

3

2

Non-hemorrhagic chronic gastric ulcer

4

Hemobilia

1

Gastroduodenitis

20

Gastric tumor

Undetectable source

1

2

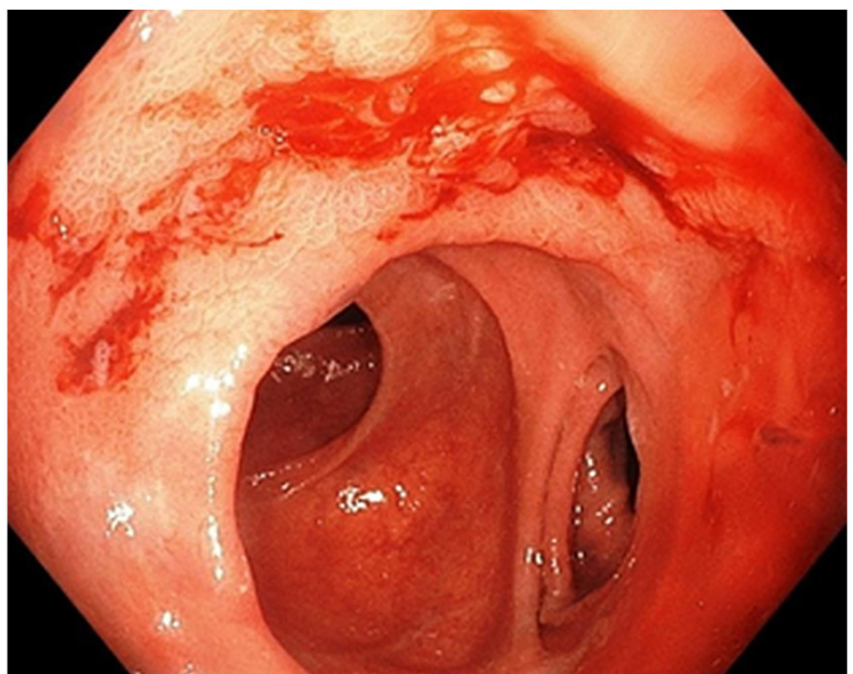

Figure 2. Upper digestive endoscopy. Ulcer (Forrest IB) of the anastomosis (gastrojejunal) mouth with 'belly bleeding' in an $H$. pylori-positive patient. H. pylori, Helicobacter pylori.

and much higher in the treatment-resistant patients $(40 \%)$ (Fig. 2).

Histological and IHC methods represented the most specific way of detecting this bacterium. In regards to all patients for whom histological and noninvasive tests were positive, IHC methods showed the presence of $H$. pylori. Moreover, IHC detected 6 more cases in which the initial noninvasive tests were negative, and the histological examination detected only 2 cases out of 6 (Figs. 3 and 4).

In 2 of the 6 cases, the IHC assessment consisted of metaplasia-associated lesions. In one case, the diagnosis of gastric carcinoma was established by IHC, and in another, the diagnosis of gastric mucosa-associated lymphoma (MALT) was established. The patient with gastric carcinoma underwent surgical operation and underwent a subtotal gastrectomy, and histological reassessment confirmed the diagnosis of carcinoma. In the case of MALT lymphoma, a total gastrectomy was performed, and the histological evaluation and IHC were performed on specimens directly from the resected piece.

Surgery for hemostasis was needed more frequently in patients with $H$. pylori-positive UGIB than in $H$. pylori-negative patients. Approximately $16 \%$ of patients with non-variceal UGIB and positive $H$. pylori results required surgery to stop the bleeding. In the group of $H$. pylori-negative patients, the need for surgery was lower; respectively $9.7 \%$ of patients underwent surgery (suturing or resection). However, distant mortality is influenced by the occurrence of bleeding, and in our study, in patients with non-variceal UGIB due to an $H$. pylori-positive lesion, it was lower compared to $H$. pylori-negative patients. We considered this difference due to the strict supervision of the therapy in the case of H. pylori-positive patients and due to the information of the 


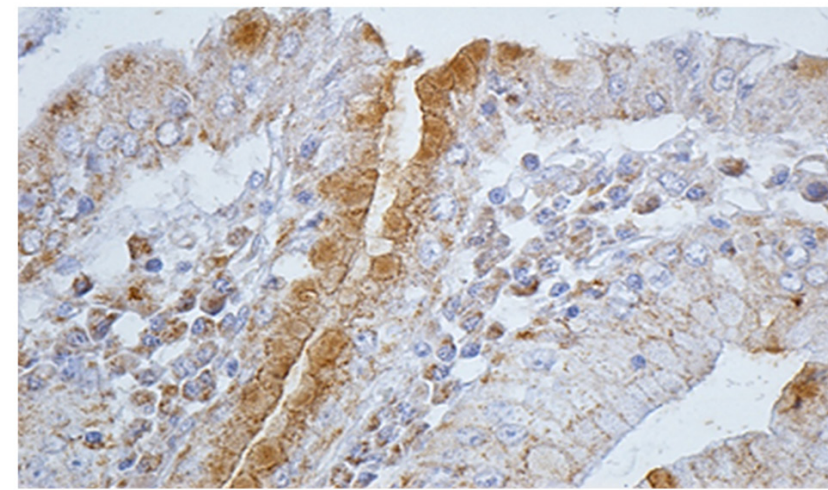

Figure 3. Immunohistochemistry exam: intense positive reaction for H. pylori. H. pylori, Helicobacter pylori.

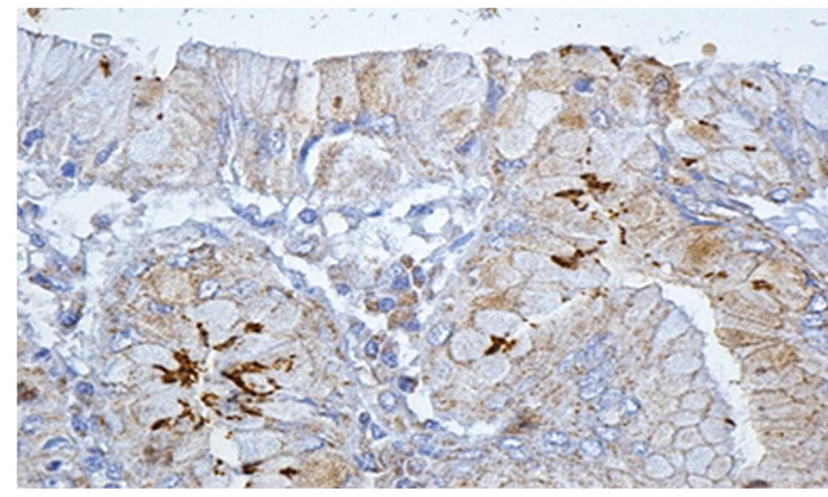

Figure 4. Immunohistochemistry exam: positive reaction for $H$. pylori. H. pylori, Helicobacter pylori.

patients about their disease and its evolution without treatment.

\section{Discussion}

In the present study, we found an increased frequency of hemorrhagic lesions among men in the group of $H$. pylori-positive patients compared to the group of $H$. pylori-negative patients (85.04 vs. $70.83 \%)$. At the same time, the frequency of hemorrhagic lesions among $H$. pylori-negative women was higher than among the $H$. pylori-positive women (29.17 vs. $15.96 \%)$. Consistent with these data, Slăvescu et al conducted a study regarding the prevalence of $H$. pylori infection in children in Romania, and found a higher prevalence among girls (9). H. pylori infection seems to act convergently with other factors, such as alcohol consumption, NSAIDs, and smoking, in favor of triggering digestive bleeding. Another hypothesis that supports these studies showed an increased frequency of antibiotic resistance of $H$. pylori in men compared to women, but dependent on the therapeutic regimen followed (10). Antibiotic resistance of $H$. pylori and poor medical education may also be factors considered in $H$. pylori-positive patients who have a history of gastric ulcer for which surgery has been performed (11) The persistence of $H$. pylori infection, despite gastric resections, still predisposes to a hemorrhagic complication (12) According to several studies, H. pylori infection in the remaining stomach appears to play an important role in the development of gastric cancer (12-14) even though the prevalence of $H$. pylori in the remaining stomach appears to be lower than that in the intact organ (11).

In the present study, the prevalence of $H$. pylori infection increased in direct proportion to age $\leq 50$ years, and then a slight decrease was found in elderly patients. The most common hemorrhagic lesions caused by $H$. pylori were in the age segment between 40 and 50 years. Thus, in this age segment, $>23 \%$ of the patients with non-variceal UGIB had gastroduodenal lesions that were directly influenced by the presence of $H$. pylori. The prevalence of hemorrhagic complications caused by $H$. pylori in the patients studied, increased sharply in the young age segment (30-50 years), then gradually decreased, reaching another peak in the age segment 70-79 years. Data from the literature report similar results $(15,16)$. In the study group, for patients $<50$ years of age, NSAIDs and $H$.pylori infection had a cumulative effect in causing hemorrhagic lesions. In contrast, after the age of 50 years, the main causes of bleeding lesions in patients with $H$. pylori-positive gastritis were the consumption of NSAIDs and treatment with antiplatelet agents or oral anticoagulants. An endoscopic study of Pilotto (17), performed in 520 patients with peptic hemorrhagic ulcer $>65$ years (mean age: 81 years), reported that $67 \%$ of gastric ulcers and $69 \%$ of duodenal ulcers were H.pylori-positive. In addition, the use of NSAIDs or aspirin, alone or in combination with $H$. pylori infection, was reported in $39 \%$ of gastric ulcers and in $25 \%$ of patients with duodenal ulcers (17). Studies do not take into account the difference between the use of NSAIDs and the use of low-dose aspirin (antiplatelet effect). $H$. pylori is associated with an increased risk of bleeding in patients under chronic treatment with AINS or antiplatelet medication (18-20). In the present study, therapies with antiplatelet agents or oral anticoagulants and $H$.pylori infection were the most frequent associations encountered in cases with active bleeding in the study group. At the same time, in elderly patients with $H$.pylori-positive gastritis who started long-term treatment with NSAIDs, oral antiplatelet agents or anticoagulants, treatment of the infection significantly reduced the risk of peptic ulcer (21-23). Patients $>50$ who are administered antiplatelet agents or oral anticoagulants, in addition to performing an upper digestive endoscopy, should be considered for a non-invasive test for the diagnosis of $H$. pylori (21). In our study, the transformation of non-hemorrhagic gastroduodenal $H$. pylori-positive lesions into hemorrhagic lesions was strongly related to alcohol consumption. We analyzed the effect of NSAID consumption associated with alcohol consumption depending on the presence of $H$. pylori and found that chronic alcohol consumption appeared to have a similar NSAID effect, so that alcohol consumption without the association with NSAIDs was considered to cause bleeding in patients with $H$. pylori-positive lesions in $\sim 14.29 \%$ of patients; $28.75 \%$ of $H$. pylori-positive patients with active bleeding at endoscopic evaluation (class IA and IB) were treated with NSAIDs and were chronic consumers of alcohol compared to patients with $H$. pylori-negative hemorrhagic lesions (12.50\% of patients with NSAIDs and alcohol). Studies that looked at the relationship between $H$. pylori infection and alcohol consumption had conflicting results. A multiple 
logistical study found that alcohol consumption and active gastritis pathology were associated with $H$. pylori infection, and active gastritis (hemorrhagic or non-hemorrhagic) was associated with chronic alcohol consumption (24). Recent studies found an increased prevalence of $H$.pylori infection in type 2 diabetes $(25,26)$, one plausible explanation being related to the changes in microbiota and low grade chronic inflammation at the level of the gastroduodenal mucosa (27). There is growing evidence of a bilateral relationship between $H$. pylori infection and chronic hepatic diseases $(28,29)$. On the one hand, the liver plays significant roles in multiple metabolic pathways, being involved in coagulation and the trophicity of gastroduodenal mucosa (30). On the other hand, chronic inflammation caused by $H$. pylori induces chronic liver fibrosis (28).

Literature data found evidence that H.pylori and NSAIDs are major causes of gastroduodenal ulcer, and an in-depth analysis of the interaction data revealed that the induction effects of ulcers for both factors were cumulative (31). Eradication of $H$. pylori in chronic NSAID users was found to decrease the incidence of ulcer disease (2). Chronic alcohol consumption appears to have a similar effect as NSAIDs, thus chronic alcohol consumption without an association with NSAIDs was considered to cause bleeding in patients with $H$. pylori-positive lesions in $\sim 14.29 \%$ of cases included in group A. In our study, $18.18 \%$ of alcohol consuming $H$. pylori-positive patients with active bleeding lesions (diagnosed endoscopically or surgically) became negative after specific treatment, while $40 \%$ of all these patients remained positive after therapy.

In the present study, $>50 \%$ of patients had $H$. pylori-positive bleeding lesions. The 30-day retest of the hemorrhagic episode in all 166 patients showed that out of the 76 initially negative patients, 6 patients became positive. These results are due to the fact that when patients have acute gastroduodenal bleeding, most diagnostic tests for $H$. pylori infection may show false-negative results (32) and the sensitivity and specificity of the fecal antigen test is $90 \%$ (2). Non-invasive testing by detecting $H$. pylori-specific antigen in feces had increased sensitivity in the diagnosis of infection in our study, so that out of 166 patients tested, a positive diagnosis was established with increased accuracy in 70 of the 76 H.pylori-positive patients. Although histological evaluation and IHC established the presence of $H$. pylori in another 6 patients, this non-invasive test had a sensitivity and specificity of over $90 \%$ in assessing the correct diagnosis. This indirect method of diagnosis is cheaper than other methods and has the advantage that it can be performed in most medical centers $(1,2)$. We found that histological examination of $H$. pylori lesions is very accurate in providing data on the degree of atrophy, metaphase or carcinoma, and the addition of IHC examination brings more information and establishes the correct diagnosis in all cases. Data from the literature show that non-invasive methods and histological evaluation of endobiopsies together establish the diagnosis of $H$. pylori infection in $>95-97 \%$ of cases $(33,34)$. In the patients in the study, the diagnosis of $H$.pylori infection was established after performing non-invasive tests and after histological evaluation in $94 \%$ of cases; this share of the diagnosis is similar to other studies. Highlighting H. pylori in the usual staining was easier in patients with acute lesions of the gastric mucosa (acute gastritis), compared to chronic (premalignant lesions: atrophic gastritis and metaplasia). Specialist studies indicate that when changes in atrophy occur in the gastric mucosa, a high percentage of endobioptic samples become negative on histological evaluation (35), and in areas with metaplasia, H. pylori is undetectable by conventional histological staining in most cases, despite serological evidence (36). In addition, the low prevalence of $H$. pylori in antral biopsy specimens with atrophic mucosa can be explained by an uneven distribution of bacterial infection resulting from $\mathrm{pH}$-increasing conditions $(37,38)$. IHC evaluation may be useful in conditions where no bacteria is visible in the usual H\&E or Giemsa staining, but there is histological evidence of inflammation; in patients with MALT from post-treatment biopsy specimens (to ensure that eradication therapy was successful) and in forms in which $H$. pylori cannot be conclusively identified (32). Routine IHC evaluation of this pathology is questionable due to the high cost and need for specialized personnel; however, it can be considered due to the accuracy of the diagnosis and the accuracy of identification of associated lesions (atrophic gastritis, metaplasia, dysplasia, carcinoma, lymphoma) (39).

Aggression and resistance of $H$. pylori to antibiotic therapy is correlated positively with an increased rate of active bleeding. Evaluation of patients with active bleeding on endoscopic examination showed that the bleeding rate was high in treatment-resistant $H$. pylori-positive patients (40\%), compared to those who were negative after specific therapy (18.18\%). In contrast, we found that the frequency of hemorrhagic lesions was much lower in those with $H$. pylori-negative lesions $(15.79 \%)$ compared to $H$. pylori-positive patients. Literature data show that before the eradication of $H$. pylori, $20-25 \%$ of patients with peptic ulcer disease developed complications such as hemorrhage, perforation or stenosis (40) and it is estimated that $\sim 1 / 3$ of $H$. pylori-positive patients with hemorrhagic ulcer will develop recurrent bleeding in the next 1-2 years in the absence of testing and eradication of $H$. pylori (41). The increased resistance of $H$. pylori to antibiotics is also correlated with the higher rate of bleeding at the distance of the hemorrhagic episode, despite PPI therapy. Endoscopic evaluation should be performed in all patients with hemorrhagic lesions for diagnostic or therapeutic purposes, but also to obtain biopsies for histological or IHC diagnosis of H. pylori (2). In our study, acute hemorrhagic lesions (gastric, duodenal ulcer or hemorrhagic gastroduodenitis) were found more frequently in the group of $H$. pylori-positive patients than in $H$. pylori-negative ones (69 vs. 43 cases). Acute hemorrhagic lesions are more common in $H$. pylori-positive patients, so acute hemorrhagic duodenal ulcer and acute hemorrhagic gastric ulcer are more common in these patients than in $H$. pylori-negative patients $(41,42)$. Chronic hemorrhagic endoscopic lesions (chronic duodenal ulcer, chronic gastric ulcer), as well as non-hemorrhagic ones, seem to be more common in $H$. pylori-negative patients. Hemorrhage may occur more frequently in the colonization phase associated with acute $H$. pylori gastritis compared to chronic lesions. At the same time, tumor lesions were much more common in $H$. pylori-positive patients ( 7 vs. 1 patient). Data from the 
literature have shown that endoscopic features have been reported to be useful tools for diagnosing $H$. pylori infection in the gastric mucosa. However, the ability to predict these characteristics can vary greatly (8).

In the present study, the need for surgery for hemostasis was more common in patients with UGIB with $H$. pylori-positive gastroduodenal lesions, compared to $H$. pylori-negative patients. Approximately $16 \%$ of patients with non-variceal and $H$. pylori-positive UGIB required surgery to stop the bleeding (16 vs. 9.7\%). Regarding the type of surgery, in the case of $H$. pylori-positive patients with UGIB, resection was needed in several cases, compared to the group of patients with $H$.pylori-negative hemorrhagic lesions. At the same time, according to other studies, postoperative complications appear more frequently in patients who undergo resection (gastrectomies, duodenopancreatectomies), compared to patients in whom suturing is performed (16,43-46). Postoperative adherences are significant causes of long term morbidity, causing episodes of abdominal pain and bowel obstruction (47).

The outcome of patients with UGIB depends very much on the need for surgery for hemostasis. The need for hemostasis surgery in the case of non-variceal UGIB is the most constant indicator of prolonged hospitalization and high financial cost, and the prophylaxis of these episodes of bleeding is the simplest modality of treatment. Thus, the presence of $H$. pylori significantly contributes to the increase in hospital stay and treatment costs in these patients.

\section{Acknowledgements}

Not applicable.

\section{Funding}

No funding was received.

\section{Availability of data and materials}

All data generated or analyzed during this study are included in this published article.

\section{Authors' contributions}

DGP, CVO, MEC, DS, CMe, MC and BN led the conception and design of this study. BS, DNF, VDB, MST, CMe MEC, $\mathrm{MD}, \mathrm{LCT}$, IDV, and BN were responsible for the data collection and analysis. CMi, DNF, VDB, MC, LCT and DS were in charge of reviewing the data and drafting the manuscript. DGP, BS, DS, CMi, MST and BN revised critical perspectives for important intellectual content. The final version was read and approved by all the authors.

\section{Ethics approval and consent to participate}

The study was conducted according to the World Medical Association Declaration of Helsinki, using a protocol approved by the local Bioethics Committee from County Emergency Clinical Hospital (Craiova, Romania). All patients previously signed an informed written consent concerning the hospitalization and research investigation.

\section{Patient consent for publication}

Not applicable.

\section{Competing interests}

The authors declare that they have no competing interests.

\section{References}

1. Obleagă CV, Vere CC, Vîlcea ID, Ciorbagiu MC, Moraru E and Mirea CS: Helicobacter pylori: Types of diseases, diagnosis, treatment and causes of therapeutic failure. J Mind Med Sci 3: 156-161, 2016.

2. Kuipers EJ, Thijs JC and Festen HP: The prevalence of Helicobacter pylori in peptic ulcer disease. Aliment Pharmacol Ther 9 (Suppl 20): S59-S69, 1995.

3. Hentschell E, Brandstätter G, Dragosics B, Hirschl AM, Nemec H, Schütze K, Taufer M and Wurzer H: Effect of ranitidine and amoxycillin plus metronidazole on the eradication of Helicobacter pylori and the recurrence of duodenal ulcer. N Engl J Med 328: 308-312, 1993.

4. Gisbert JP, Khorrami S, Carballo F, Calvet X, Gene E and Dominguez-Munoz E: Meta-analysis: Helicobacter pylori eradication therapy vs. antisecretory non-eradication therapy for the prevention of recurrent bleeding from peptic ulcer. Aliment Pharmacol Ther 19: 617-629, 2004.

5. Guo CG, Cheung KS, Zhang F, Chan EW, Chen L, Wong ICK and Leung WK: Delay in retreatment of Helicobacter pylori infection increases risk of upper gastrointestinal bleeding. Clin Gastroenterol Hepatol 19: 314-322.e2, 2021.

6. Gisbert JP and Abraira V: Accuracy of Helicobacter pylori diagnostic tests in patients with bleeding peptic ulcer: A systematic review and meta-analysis. Am J Gastroenterol 101: 848-863, 2006.

7. Stolte $M$ and Meining A: The updated Sydney system: Classification and grading of gastritis as the basis of diagnosis and treatment. Can J Gastroenterol 15: 591-598, 2001.

8. Mao T, Wang Y, Yin F, Zhao Q, Yang L, Ding X and Tian Z: Association of endoscopic features of gastric mucosa with Helicobacter pylori infection in Chinese patients. Gastroenterol Res Pract 2016: 6539639, 2016.

9. Slăvescu KC, Șarban C, Pîrvan A, Gheban D, Mărgescu C and Miu N: Prevalence of Helicobacter pylori infection in children with gastritis and peptic ulcer disease in northwestern and central Romania. Med Pharmacy Rep 85: 457-462, 2012.

10. Megraud F: H. pylori antibiotic resistance: Prevalence, importance and advances in testing. Gut 53: 1374-1384, 2004.

11. Park S and Chun HJ: Helicobacter pylori infection following partial gastrectomy for gastric cancer. World J Gastroenterol 20: 2765-2770, 2014.

12. Testerman TL and Morris J: Beyond the stomach: An updated view of Helicobacter pylori pathogenesis, diagnosis, and treatment. World J Gastroenterol 20: 12781-12808, 2014.

13. Wroblewski LE, Peek RM Jr and Wilson KT: Helicobacter pylori and gastric cancer: Factors that modulate disease risk. Clin Microbiol Rev 23: 713-739, 2010.

14. Conteduca V, Sansonno D, Lauletta G, Russi S, Ingravallo G and Dammacco F: H. pylori infection and gastric cancer: State of the art (Review). Int J Oncol 42: 5-18, 2013.

15. Alberto $\mathrm{P}$ and Franceschi M: Helicobacter pylori infection in older people. World J Gastroenterol 20: 6364-6373, 2014.

16. Constantin VD, Paun S, Ciofoaia VV, Budu V and Socea B: Multimodal management of upper gastrointestinal bleeding caused by stress gastropathy. J Gastrointestin Liver Dis 18: 279-284, 2009.

17. Pilotto A: Aging and upper gastrointestinal disorders. Best Pract Res Clin Gastroenterol 18 (Suppl): S73-S81, 2004.

18. Venerito M, Schneider C, Costanzo R, Breja R, Röhl FW and Malfertheiner P: Contribution of Helicobacter pylori infection to the risk of peptic ulcer bleeding in patients on nonsteroidal anti-inflammatory drugs, antiplatelet agents, anticoagulants, corticosteroids and selective serotonin reuptake inhibitors. Aliment Pharmacol Ther 47: 1464-1471, 2018.

19. Pilotto A and Franceschi M: Helicobacter pylori infection in older people. World J Gastroenterol 20: 6364-6373, 2014. 
20. Lanas A, Dumonceau JM, Hunt RH, Fujishiro M, Scheiman JM Gralnek IM, Campbell HE, Rostom A, Villanueva C and Sung JJY: Non-variceal upper gastrointestinal bleeding. Nat Rev Dis Primers 4: 18020, 2018.

21. Pilotto A, Franceschi M, Leandro G, Paris F, Cascavilla L, Longo MG, Niro V, Andriulli A, Scarcelli C and Di Mario F: Proton-pump inhibitors reduce the risk of uncomplicated peptic ulcer in elderly either acute or chronic users of aspirin/non-steroidal anti-inflammatory drugs. Aliment Pharmacol Ther 20: 1091-1097, 2004.

22. Narayanan M, Reddy KM and Marsicano E: Peptic ulcer disease and Helicobacter pylori infection. Mo Med 115: 219-224, 2018.

23. Zapata-Colindres JC, Zepeda-Gómez S, Montaño-Loza A Vázquez-Ballesteros E, De Jesús Villalobos J and Valdovinos-Andraca F: The association of Helicobacter pylori infection and nonsteroidal anti-inflammatory drugs in peptic ulcer disease. Can J Gastroenterol 20: 277-280, 2006.

24. Zhang L, Eslick GD, Xia HH, Wu C, Phung N and Talley NJ: Relationship between alcohol consumption and active Helicobacter pylori infection. Alcohol Alcohol 45: 89-94, 2010.

25. Hosseininasab Nodoushan SA and Nabavi A: The interaction of Helicobacter pylori infection and type 2 diabetes mellitus. Adv Biomed Res 8: 15, 2019.

26. He C, Yang $\mathrm{Z}$ and Lu NH: Helicobacter pylori infection and diabetes: Is it a myth or fact? World J Gastroenterol 20 : 4607-4617, 2018

27. Suceveanu AI, Stoian AP, Parepa I, Voinea C, Hainarosie R, Manuc D, Nitipir C, Mazilu L and Suceveanu AP: Gut microbiota patterns in obese and type 2 diabetes (T2D) patients from romanian black sea coast region. Rev Chim 69: 2260-2267, 2018

28. Okushin K, Tsutsumi T, Ikeuchi K, Kado A,Enooku K, Fujinaga H Moriya K, Yotsuyanagi H and Koike K: Helicobacter pylori infection and liver diseases: Epidemiology and insights into pathogenesis. World J Gastroenterol 24: 3617-3625, 2018.

29. Tsai CE, Liang CM, Lee CH, Kuo YH, Wu KL, Chiu YC, Tai WC and Chuah SK: First-line Helicobacter pylori eradication among patients with chronic liver diseases in Taiwan. Kaohsiung J Med Sci 32: 397-402, 2016.

30. Gheorghe G, Stoian AP, Gaman M, Socea B, Neagu TP Stanescu AMA, Bratu OG, Mischianu DLD, Suceveanu AI and Diaconu CC: The Benefits and Risks of Antioxidant Treatment in Liver Diseases. Rev Chim 70: 651, 2019.

31. Huan JQ, Sridhar S and Hunt RH: Role of Helicobacter pylori infection and nonsteroidal anti-inflammatory drugs in peptic-ulcer disease: A meta-analysis. Lancet 359: 14-22, 2002.

32. Braden B: Diagnosis of Helicobacter pylori infection. BMJ 344: $828,2012$.

33. Lee JY and Kim N: Diagnosis of Helicobacter pylori by invasive test: Histology. Ann Transl Med 3: 10, 2015.

34. Alius C, Tudor C, Badiu CD, Dascalu AM, Smarandache CG, Sabau AD, Tanasescu C, Balasescu SA and Serban D: Indocyanine green-enhanced colorectal surgery-between being superfluous and being a game-changer. Diagnostics (Basel) 10: $742,2020$.
35. Kokkola A, Rautelin H, Puolakkainen P, Sipponen P, Färkkilä M, Haapiainen R and Kosunen TU: Diagnosis of Helicobacter pylori infection in patients with atrophic gastritis: Comparison of histology, 13C-urea breath test, and serology. Scand J Gastroenterol 35: 138-141, 2000.

36. Loffeld RJ, Stobberingh E, Flendrig JA and Arends JW: Helicobacter pylori in gastric biopsy specimens. Comparison of culture, modified giemsa stain, and immunohistochemistry. A retrospective study. J Pathol 165: 69-73, 1991.

37. Testoni PA, Colombo E, Cattani L, Longhi M, Bagnolo F, Lella F, Buizza M and Scelsi R: Helicobacter pylori serology in chronic gastritis with antral atrophy and negative histology for Helicobacter-like organisms. J Clin Gastroenterol 22: 182-185, 1996.

38. Kang HY, Kim N, Park YS, Hwang JH, Kim JW, Jeong SH, Lee DH, Jung HC and Song IS: Progression of atrophic gastritis and intestinal metaplasia drives Helicobacter pylori out of the gastric mucosa. Dig Dis Sci 51: 2310-2315, 2006.

39. Park YH and Kim N: Review of atrophic gastritis and intestinal metaplasia as a premalignant lesion of gastric cancer. J Cancer Prev 20: 25-40, 2015.

40. Penston JG: A decade of experience with long-term continuous treatment of peptic ulcers with $\mathrm{H} 2$-receptor antagonists. Aliment Pharmacol Ther 7 (Suppl 2): S27-S33, 1993.

41. Laine LA: Helicobacter pylori and complicated ulcer disease. Am J Med 100 (Suppl): S52-S59, 1996.

42. Campuzano-Maya G: Hematologic manifestations of Helicobacter pylori infection. World J Gastroenterol 20: 12818-12838, 2014

43. Obleagă CV, Vere CC, Pătraşcu AM, Moraru E, Crafciuc AV, Foarfă MC, Mogoantă SŞ, Streba CT, Bondari S, Paitici Ş et al: Severe upper gastrointestinal bleeding determined by a gastric lymphoma associated with Helicobacter pylori-positive atrophic gastritis. Rom J Morphol Embryol 58: 611-617, 2017.

44. Obleagă CV, Vere CC, Mogoanţa S, Firut C, Meșina C, Ciorbagiu MC, Mirea CS and Vîlcea ID: Upper gastrointestinal bleeding-initial manifestation of pancreatic head carcinoma. Curr Health Sci J 43: 236-240, 2017.

45. Serban D, Vancea G, Balasescu SA, Socea B, Tudor C and Dascalu AM: Informed consent in all surgical specialties: From legal obligation to patient satisfaction. Rom J Leg Med 28: 317-321, 2020.

46. Constantin VD, Socea B, Sireteanu G and Popa F: Epidemiological aspects and risk factors in the outcome of variceal eso-gastric bleeding at cirrhosis patients. J Appl Quant Meth 3: 316-324, 2008.

47. Fometescu SG, Costache M, Coveney A, Oprescu SM, Serban D and Savlovschi C: Peritoneal fibrinolytic activity and adhesiogenesis. Chirurgia (Bucur) 108: 331-340, 2013. International (CC BY-NC-ND 4.0) License. 